\title{
Axial-vector form factors for the low lying octet baryons in the chiral quark constituent model
}

\author{
Harleen Dahiya \\ Department of Physics, \\ Dr. B.R. Ambedkar National Institute of Technology, \\ Jalandhar, 144011, India \\ Monika Randhawa \\ University Institute of Engineering and Technology, \\ Panjab University, Chandigarh, India
}

\begin{abstract}
We have calculated the axial-vector form factors of the low lying octet baryons $(N, \Sigma, \Xi$ and $\Lambda)$ in the chiral constituent quark model $(\chi \mathrm{CQM})$. In particular, we have studied the implications of chiral symmetry breaking and SU(3) symmetry breaking for the singlet $\left(g_{A}^{0}\right)$ and non-singlet $\left(g_{A}^{3}\right.$ and $g_{A}^{8}$ ) axial-vector coupling constants expressed as combinations of the spin polarizations at zero momentum transfer. The conventional dipole form of parametrization has been used to analyse the $Q^{2}$ dependence of the axial-vector form factors $\left(G_{A}^{0}\left(Q^{2}\right), G_{A}^{3}\left(Q^{2}\right)\right.$ and $\left.G_{A}^{8}\left(Q^{2}\right)\right)$. The total strange singlet and non-singlet contents $\left(G_{s}^{0}\left(Q^{2}\right), G_{s}^{3}\left(Q^{2}\right)\right.$ and $\left.G_{s}^{8}\left(Q^{2}\right)\right)$ of the nucleon determining the strange quark contribution to the nucleon spin $(\Delta s)$ have also been discussed.
\end{abstract}




\section{INTRODUCTION}

The internal structure of the baryons has been extensively studied ever since the measurements of polarized structure functions of proton in the deep inelastic scattering (DIS) experiments [1-4]. These experiments have provided the first evidence that the valence quarks of proton carry only a small fraction of its spin and the decomposition of the proton's spin still remains to be a major unresolved issue in high energy spin physics. Form factors parameterized from the electromagnetic current operator as well as the isovector axial-vector current operator are important in hadron physics as they provide a deep insight in understanding the internal structure. The electromagnetic Dirac and Pauli form factors are well know over a wide region of momentum transfer squared $Q^{2}$, however, the study of the axial-vector form factors has been rather limited. The measured first moment is related to the combinations of the axial-vector coupling constants which are combinations of the spin polarizations, $\Delta u, \Delta d$ and $\Delta s$. For example,

$$
\Gamma_{1}^{p}\left(Q^{2}\right)=\int_{0}^{1} g_{1}^{p}\left(x, Q^{2}\right) d x=\frac{C_{s}\left(Q^{2}\right)}{9} g_{A}^{0}+\frac{C_{n s}\left(Q^{2}\right)}{12} g_{A}^{3}+\frac{C_{n s}\left(Q^{2}\right)}{36} g_{A}^{8} .
$$

Here $C_{s}$ and $C_{n s}$ are the flavor singlet and non-singlet Wilson coefficients calculable from perturbative QCD. The quantity $g_{A}^{0}$ corresponds to the flavor singlet component related to the total quark spin content $\Delta \Sigma$ whereas $g_{A}^{3}$ and $g_{A}^{8}$ correspond to the flavor non-singlet components usually obtained from the neutron $\beta$-decay and the semi-leptonic weak decays of hyperons respectively. These axial-vector coupling constants can be related to certain well known sum rules such as Bjorken sum rule (BSR) [5] and Ellis-Jaffe sum rule (EJSR) [6] and derived within Quantum Chromodynamics (QCD) using operator product expansion, renormalization group invariance and isospin conservation in the DIS.

Recently, experiments measuring electromagnetic and weak form factors from the elastic scattering of electrons, for example, SAMPLE at MIT-Bates [7], G0 at JLab [8], PVA4 at MAMI [9] and HAPPEX at JLab [10] have given indications of strangeness contribution in the nucleon. These experiments have provided considerable insight on the role played by strange quarks in the charge, current and spin structure of the nucleon. The nucleon axial coupling constant $g_{A}^{3}$ has received much attention in the past and has been determined precisely from nuclear $\beta$-decay [11]. It corresponds to the value of the axial form factor at zero-momentum transfer $\left(Q^{2}=-q^{2}=0\right)$. It is one of the fundamental parameter to test the chiral symmetry breaking effects and thereby determine the spin structure of the 
nucleon. Our information about the other low lying octet baryon axial-vector form factors from experiment is also rather limited because it is difficult to measure the hyperon properties experimentally due to their short lifetimes. Even though there has been considerable progress in the past few years to determine the $Q^{2}$ dependence of axial form factors experimentally, there is no consensus regarding the various mechanisms which can contribute to it. Experiments involving elastic scattering of neutrinos and antineutrinos [12, 13] and the pion electro-production on the proton [14] have explored $Q^{2}$ dependence of axial form factors in the past and they point out the need for additional refined data. More recently, there has been considerable refinement to measure the $Q^{2}$ dependence of the axial-vector form factor of the nucleon in the higher-energy Minerva experiment at Fermilab [15].

The theoretical knowledge in this regard has been rather limited because of confinement and it is still a big challenge to perform the calculations from the first principles of QCD. Even though some lattice QCD calculations of the axial charge and form factors of the nucleon have been performed [16] but still a lot of refinements need to be done. The broader question of axial charge, axial form factors and the strange quark contribution to the axial form factors of the nucleon has also been discussed by several authors in other models recently [17]. In addition to this, the partial conservation of axial-vector current (PCAC) also provide important constraints on the axial exchange currents to describe the non-valence degrees of freedom in the nucleon [18 20]. One of the most successful nonperturbative approach which finds its application for the quantities discussed above is the chiral constituent quark model $(\chi \mathrm{CQM})[21$. The basic idea is based on the possibility that chiral symmetry breaking takes place at a distance scale much smaller than the confinement scale. The $\chi$ CQM uses the effective interaction Lagrangian approach of the strong interactions where the effective degrees of freedom are the valence quarks and the internal Goldstone bosons (GBs) which are coupled to the valence quarks $[22-25]$. The $\chi$ CQM successfully explains the "proton spin problem" [25], magnetic moments of octet and decuplet baryons including their transitions [26], account for the violation of Gottfried Sum Rule [27] and Coleman-Glashow sum rule, hyperon $\beta$ decay parameters [28], strangeness content in the nucleon [29], magnetic moments of $\frac{1}{2}^{-}$octet baryon resonances [30], magnetic moments of $\frac{1}{2}^{-}$and $\frac{3}{2}^{-} \Lambda$ resonances [31], charge radii [32], quadrupole moment [33], etc.. The model is successfully extended to predict the important role played by the small intrinsic charm content in the nucleon spin in the $\mathrm{SU}(4) \chi \mathrm{CQM}\left[34\right.$ and to calculate the magnetic moment and charge radii of spin $\frac{1}{2}^{+}$ 
and spin $\frac{3}{2}^{+}$charm baryons including their radiative decays [35, 36]. The $\chi$ CQM provides a simultaneous unique information on the flavor and spin structure of the baryons including the heavy baryons. In view of the above developments in the $\chi \mathrm{CQM}$, it become desirable to extend the model to calculate the axial-vector form factors of the low-lying octet baryons. It is widely recognized that a knowledge about the axial-vector form factors of the baryons in general and the strangeness content of the nucleon in particular would undoubtedly provide vital clues to the nonperturbative aspects of QCD.

The purpose of the present communication is to determine the axial-vector form factors of the low lying octet baryons in the chiral constituent quark model ( $\chi \mathrm{CQM})$. In particular, we would like to phenomenologically estimate the quantities affected by chiral symmetry breaking and $\mathrm{SU}(3)$ symmetry breaking. We begin by computing the static properties of the axial-vector current. The singlet $\left(g_{A}^{0}\right)$ and non-singlet $\left(g_{A}^{3}\right.$ and $\left.g_{A}^{8}\right)$ axial-vector coupling constants expressed as combinations of the spin polarizations at zero momentum transfer have been investigated for the case of $N, \Sigma, \Xi$ and $\Lambda$ baryons. Further, it would be significant to analyse the $Q^{2}$ dependence of the axial-vector form factors $\left(G_{A}^{0}\left(Q^{2}\right), G_{A}^{3}\left(Q^{2}\right)\right.$ and $\left.G_{A}^{8}\left(Q^{2}\right)\right)$ as well as their explicit flavor contributions $\left(G_{A}^{u}\left(Q^{2}\right), G_{A}^{d}\left(Q^{2}\right)\right.$ and $\left.G_{A}^{s}\left(Q^{2}\right)\right)$ by using a conventional dipole form of parametrization. Furthermore, it would be interesting to extend the calculations to predict the total strange singlet and non-singlet contents $\left(G_{s}^{0}\left(Q^{2}\right), G_{s}^{3}\left(Q^{2}\right)\right.$ and $\left.G_{s}^{8}\left(Q^{2}\right)\right)$ of the nucleon and determine the strange quark contribution to the nucleon spin $(\Delta s)$. The results can be compared with the recent available experimental observations.

\section{CHIRAL CONSTITUENT QUARK MODEL}

The key to understand the structure of the baryons, in the $\chi \mathrm{CQM}$ formalism [22], is the fluctuation process

$$
q^{ \pm} \rightarrow \mathrm{GB}+q^{\prime \mp} \rightarrow\left(q \bar{q}^{\prime}\right)+q^{\prime} \mp
$$

where GB represents the Goldstone boson and $q \bar{q}^{\prime}+q^{\prime}$ constitute the "quark sea" [22, 23, 25]. The effective Lagrangian describing the interaction between quarks and a nonet of GBs, can be expressed as

$$
\mathcal{L}=c_{8} \overline{\mathbf{q}}\left(\Phi+\zeta \frac{\eta^{\prime}}{\sqrt{3}} I\right) \mathbf{q}=c_{8} \overline{\mathbf{q}}\left(\Phi^{\prime}\right) \mathbf{q},
$$

where $\zeta=c_{1} / c_{8}, c_{1}$ and $c_{8}$ are the coupling constants for the singlet and octet GBs, respectively, $I$ is the $3 \times 3$ identity matrix. The matrix $\mathbf{q}$ and the GB field can be expressed in 
terms of the GBs and their transition probabilities as

$$
\mathbf{q}=\left(\begin{array}{l}
u \\
d \\
s
\end{array}\right), \quad \Phi^{\prime}=\left(\begin{array}{ccc}
\frac{\pi^{o}}{\sqrt{2}}+\beta \frac{\eta}{\sqrt{6}}+\zeta \frac{\eta^{\prime}}{\sqrt{3}} & \pi^{+} & \alpha K^{+} \\
\pi^{-} & -\frac{\pi^{o}}{\sqrt{2}}+\beta \frac{\eta}{\sqrt{6}}+\zeta \frac{\eta^{\prime}}{\sqrt{3}} & \alpha K^{o} \\
\alpha K^{-} & \alpha \bar{K}^{o} & -\beta \frac{2 \eta}{\sqrt{6}}+\zeta \frac{\eta^{\prime}}{\sqrt{3}}
\end{array}\right) .
$$

If the parameter $a\left(=\left|c_{8}\right|^{2}\right)$ denotes the transition probability of chiral fluctuation of the splitting $u(d) \rightarrow d(u)+\pi^{+(-)}$, then $\alpha^{2} a, \beta^{2} a$ and $\zeta^{2} a$ respectively, denote the probabilities of transitions of $u(d) \rightarrow s+K^{+(0)}, u(d, s) \rightarrow u(d, s)+\eta$, and $u(d, s) \rightarrow u(d, s)+\eta^{\prime}[22$, 23]. These parameters provide the basis to understand the extent to which the quark sea contributes to the structure of the baryon. The symmetry breaking parameter $a$ is introduced by considering nondegenerate quark masses $M_{s}>M_{u, d}$, the parameters $\alpha$ and $\beta$ are introduced by considering nondegenerate GB masses $M_{K, \eta}>M_{\pi}$ and finally the parameter $\zeta$ is introduced by considering $M_{\eta^{\prime}}>M_{K, \eta}$. Since the quark contributions scale as $\frac{1}{M_{q}^{2}}$, a hierarchy for the probabilities can be obtained as

$$
a>a \alpha^{2} \geq a \beta^{2}>a \zeta^{2} .
$$

Before proceeding further to calculate the axial-vector form factors, we briefly discuss the calculation of the spin structure of the baryons. Following references [22, 23], the quark spin polarization can be defined as

$$
\Delta q=q^{+}-q^{-}
$$

where $q^{ \pm}$can be calculated from the spin structure of a baryon

$$
\hat{B} \equiv\langle B|\mathcal{N}| B\rangle=\left\langle B\left|q^{+} q^{-}\right| B\right\rangle
$$

Here $|B\rangle$ is the baryon wave function and $\mathcal{N}=q^{+} q^{-}$is the number operator measuring the sum of the quark numbers with spin up or down, for example,

$$
q^{+} q^{-}=\sum_{q=u, d, s}\left(n_{q^{+}} q^{+}+n_{q^{-}} q^{-}\right)=n_{u^{+}} u^{+}+n_{u^{-}} u^{-}+n_{d^{+}} d^{+}+n_{d^{-}} d^{-}+n_{s^{+}} s^{+}+n_{s^{-}} s^{-},
$$

with the coefficients of the $q^{ \pm}$giving the number of $q^{ \pm}$quarks. The contributions of the quark sea coming from the fluctuation process in Eq. (2) can be calculated by substituting for every constituent quark

$$
q^{ \pm} \rightarrow \sum P_{q} q^{ \pm}+\left|\psi\left(q^{ \pm}\right)\right|^{2}
$$


where the transition probability of the emission of a GB from any of the $q$ quark $\left(\sum P_{q}\right)$ and the transition probability of the $q^{ \pm}$quark $\left(\left|\psi\left(q^{ \pm}\right)\right|^{2}\right)$ can be calculated from the Lagrangian. They are expressed as

$$
\begin{aligned}
& \sum P_{u}=a\left(\frac{9+\beta^{2}+2 \zeta^{2}}{6}+\alpha^{2}\right) \quad \text { and } \quad\left|\psi\left(u^{ \pm}\right)\right|^{2}=\frac{a}{6}\left(3+\beta^{2}+2 \zeta^{2}\right) u^{\mp}+a d^{\mp}+a \alpha^{2} s^{\mp}, \\
& \sum P_{d}=a\left(\frac{9+\beta^{2}+2 \zeta^{2}}{6}+\alpha^{2}\right) \text { and }\left|\psi\left(d^{ \pm}\right)\right|^{2}=a u^{\mp}+\frac{a}{6}\left(3+\beta^{2}+2 \zeta^{2}\right) d^{\mp}+a \alpha^{2} s^{\mp}, \\
& \sum P_{s}=a\left(\frac{2 \beta^{2}+\zeta^{2}}{3}+2 \alpha^{2}\right) \quad \text { and }\left|\psi\left(s^{ \pm}\right)\right|^{2}=a \alpha^{2} u^{\mp}+a \alpha^{2} d^{\mp}+\frac{a}{3}\left(2 \beta^{2}+\zeta^{2}\right) s^{\mp} .
\end{aligned}
$$

Spin-spin forces, known to be compatible [37 39] with the $\chi \mathrm{QM}$, generate configuration mixing [40 42] for the octet baryons which effectively leads to modification of the spin distribution functions [25]. The general configuration mixing generated by the spin-spin forces has been discussed in the case of octet baryons [40, 42, 43]. However, it is adequate [25, 42, 44, 45] to consider the "mixed" octet with mixing only between $\left|56,0^{+}\right\rangle_{N=0}$ and the $\left|70,0^{+}\right\rangle_{N=2}$ states, for example,

$$
|B\rangle \equiv\left|8, \frac{1^{+}}{2}\right\rangle=\cos \phi\left|56,0^{+}\right\rangle_{N=0}+\sin \phi\left|70,0^{+}\right\rangle_{N=2},
$$

where $\phi$ represents the $|56\rangle-|70\rangle$ mixing and

$$
\begin{aligned}
\left|56,0^{+}\right\rangle_{N=0} & =\frac{1}{\sqrt{2}}\left(\chi^{\prime} \phi^{\prime}+\chi^{\prime \prime} \phi^{\prime \prime}\right) \psi^{s}\left(0^{+}\right), \\
\left|70,0^{+}\right\rangle_{N=2} & =\frac{1}{2}\left[\left(\phi^{\prime} \chi^{\prime \prime}+\phi^{\prime \prime} \chi^{\prime}\right) \psi^{\prime}\left(0^{+}\right)+\left(\phi^{\prime} \chi^{\prime}-\phi^{\prime \prime} \chi^{\prime \prime}\right) \psi^{\prime \prime}\left(0^{+}\right)\right] .
\end{aligned}
$$

In general, the isospin wave functions for the octet baryons $(N, \Sigma, \Xi)$ of the type $B(x x y)$ are given as

$$
\phi_{B}^{\prime}=\frac{1}{\sqrt{2}}(x y x-y x x), \quad \phi_{B}^{\prime \prime}=\frac{1}{\sqrt{6}}(2 x x y-x y x-y x x),
$$

whereas for $\Lambda(u d s)$ they are given as

$$
\phi_{\Lambda}^{\prime}=\frac{1}{2 \sqrt{3}}(u s d+s d u-s u d-d s u-2 u d s-2 d u s), \quad \phi_{\Lambda}^{\prime \prime}=\frac{1}{2}(s u d+u s d-s d u-d s u) .
$$

The spin wave functions are expressed as

$$
\chi^{\prime}=\frac{1}{\sqrt{2}}(\uparrow \downarrow \uparrow-\downarrow \uparrow \uparrow), \quad \chi^{\prime \prime}=\frac{1}{\sqrt{6}}(2 \uparrow \uparrow \downarrow-\uparrow \downarrow \uparrow-\downarrow \uparrow \uparrow) .
$$


For the definition of the spatial wave functions $\left(\psi^{s}, \psi^{\prime}, \psi^{\prime \prime}\right)$ as well as the definitions of the overlap integrals, we refer the reader to reference [46].

The quark polarizations can be calculated from the spin structure of a given baryon. Using Eqs. (7) and (10) of the text, the spin structure of a baryon in the "mixed" octet is given as

$$
\hat{B} \equiv\langle B|\mathcal{N}| B\rangle=\cos ^{2} \phi\left\langle 56,0^{+}|\mathcal{N}| 56,0^{+}\right\rangle_{B}+\sin ^{2} \phi\left\langle 70,0^{+}|\mathcal{N}| 70,0^{+}\right\rangle_{B}
$$

For the case of $N, \Sigma, \Xi$ and $\Lambda$, using Eqs. (11) and (12), we have

$$
\begin{aligned}
\left\langle 56,0^{+}|\mathcal{N}| 56,0^{+}\right\rangle_{N} & =\frac{5}{3} u_{+}+\frac{1}{3} u_{-}+\frac{1}{3} d_{+}+\frac{2}{3} d_{-}, \\
\left\langle 70,0^{+}|\mathcal{N}| 70,0^{+}\right\rangle_{N} & =\frac{4}{3} u_{+}+\frac{2}{3} u_{-}+\frac{2}{3} d_{+}+\frac{1}{3} d_{-}, \\
\left\langle 56,0^{+}|\mathcal{N}| 56,0^{+}\right\rangle_{\Sigma} & =\frac{5}{3} u_{+}+\frac{1}{3} u_{-}+\frac{1}{3} s_{+}+\frac{2}{3} s_{-}, \\
\left\langle 70,0^{+}|\mathcal{N}| 70,0^{+}\right\rangle_{\Sigma} & =\frac{4}{3} u_{+}+\frac{2}{3} u_{-}+\frac{2}{3} s_{+}+\frac{1}{3} s_{-}, \\
\left\langle 56,0^{+}|\mathcal{N}| 56,0^{+}\right\rangle_{\Xi} & =\frac{5}{3} s_{+}+\frac{1}{3} s_{-}+\frac{1}{3} u_{+}+\frac{2}{3} u_{-}, \\
\left\langle 70,0^{+}|\mathcal{N}| 70,0^{+}\right\rangle_{\Xi} & =\frac{4}{3} s_{+}+\frac{2}{3} s_{-}+\frac{2}{3} u_{+}+\frac{1}{3} u_{-},
\end{aligned}
$$

and

$$
\begin{aligned}
\left\langle 56,0^{+}|\mathcal{N}| 56,0^{+}\right\rangle_{\Lambda} & =\frac{1}{2} u_{+}+\frac{1}{2} u_{-}+\frac{1}{2} d_{+}+\frac{1}{2} d_{-}+1 s_{+}+0 s_{-}, \\
\left\langle 70,0^{+}|\mathcal{N}| 70,0^{+}\right\rangle_{\Lambda} & =\frac{2}{3} u_{+}+\frac{1}{3} u_{-}+\frac{2}{3} d_{+}+\frac{1}{3} d_{-}+\frac{2}{3} s_{+}+\frac{1}{3} s_{-},
\end{aligned}
$$

respectively. Sea contributions can be included by using Eq. (9) and the results have been presented in Table II. A closer look at the expressions of these quantities reveals that the constant factors represent the Naive Quark Model (NQM) results which do not include the effects of chiral symmetry breaking. On the other hand, the factors with transition probability a represent the contribution from the "quark sea" in general (with or without $\mathrm{SU}(3)$ symmetry breaking). 


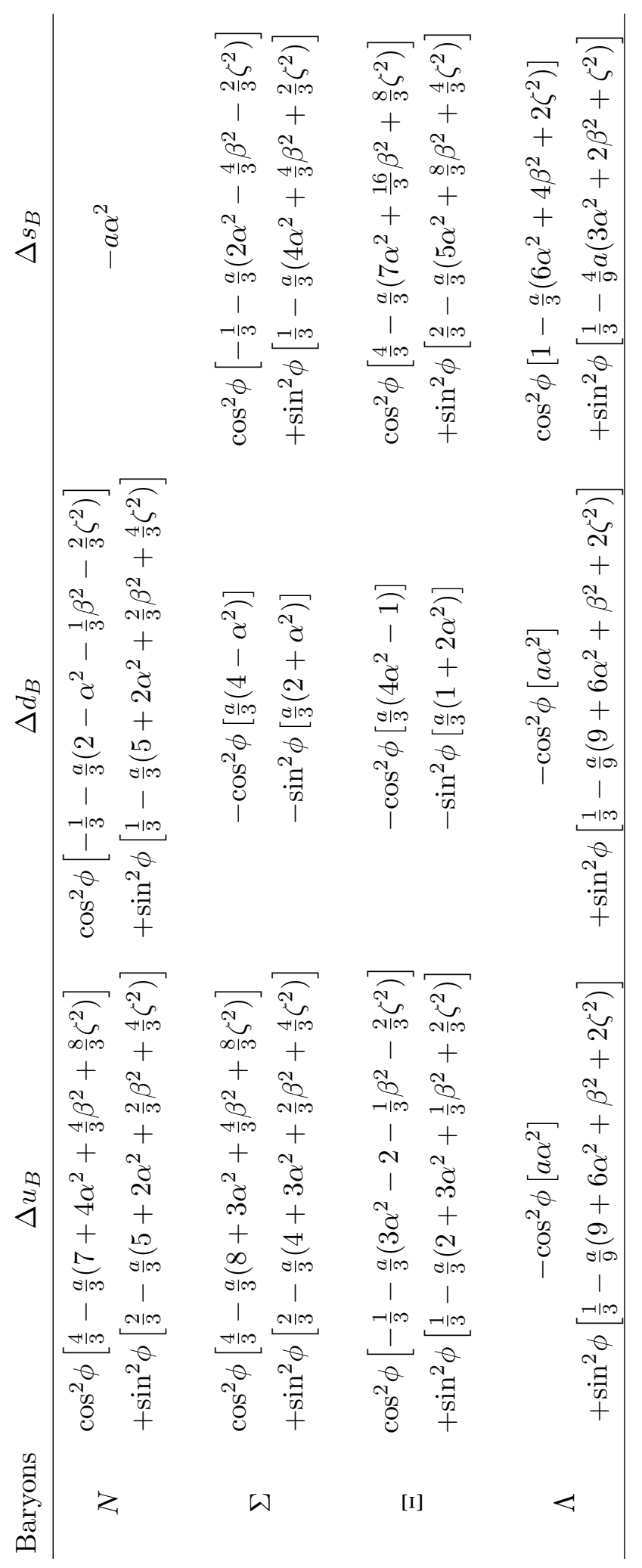

TABLE I. The quark spin polarizations for the octet baryons in the $\chi$ CQM. 


\section{AXIAL VECTOR FORM FACTORS}

The axial-vector form factors can be expressed in terms of the axial-vector current $A^{\mu, a}$ defined as $\overline{\mathbf{q}} \gamma^{\mu} \gamma_{5} \frac{\lambda^{a}}{2} \mathbf{q}$ through the following matrix elements

$$
\left\langle B\left(p^{\prime}\right)\left|A^{\mu, a}\right| B(p)\right\rangle=\bar{u}\left(p^{\prime}\right)\left[\gamma^{\mu} \gamma_{5} G_{A}^{i}\left(Q^{2}\right)+\frac{q^{\mu}}{2 M_{B}} \gamma_{5} G_{P}^{i}\left(Q^{2}\right)\right] u(p)
$$

where $M_{B}$ is the baryon mass, $u(p)\left(\bar{u}\left(p^{\prime}\right)\right)$ are the Dirac spinors of the initial (final) baryon states, respectively. The four momenta transfer is given as $Q^{2}=-q^{2}$, where $q \equiv p-p^{\prime}$. Here, $\lambda^{a}(a=1,2, . .8)$ are the Gell-Mann matrices of SU(3) describing the flavor structure of

the 3 light quarks. It is often convenient to introduce the unit matrix $\lambda^{0}\left(=\sqrt{\frac{2}{3}} I\right)$ in addition to these matrices. In the present context. we shall need only the matrices having diagonal representation corresponding to the flavor singlet current $(a=0)$, isovector current $(a=3)$ and hypercharge axial current $(a=8)$ [19]. The functions $G_{A}^{i}\left(Q^{2}\right)$ and $G_{P}^{i}\left(Q^{2}\right)(i=0,3,8)$ are the axial and induced pseudoscalar form factors respectively. We will ignore the induced pseudoscalar form factors as they not relevant to the present work.

In general, the axial-vector matrix elements have implications for spin structure [25, 28]. In order to calculate the axial charge as one of the important static property of the form factors at zero momentum transfer, the singlet and non-singlet combinations of the spin structure can be related to the weak couplings and can be expressed in terms of the spin polarizations defined in the above section. We have

$$
\begin{aligned}
& g_{A, B}^{0}=\left\langle B\left|u^{+} u^{-}+d^{+} d^{-}+s^{+} s^{-}\right| B\right\rangle=\Delta u_{B}+\Delta d_{B}+\Delta s_{B}, \\
& g_{A, B}^{3}=\left\langle B\left|u^{+} u^{-}-d^{+} d^{-}\right| B\right\rangle=\Delta u_{B}-\Delta d_{B}, \\
& g_{A, B}^{8}=\left\langle B\left|u^{+} u^{-}+d^{+} d^{-}+2 s^{+} s^{-}\right| B\right\rangle=\Delta u_{B}+\Delta d_{B}-2 \Delta s_{B} .
\end{aligned}
$$

The axial coupling constants $g_{A, B}^{3}$ and $g_{A, B}^{8}$ basically correspond to the BSR [5] and the EJSR [6]. The axial coupling constant related to the total quark spin content $g_{A, B}^{0}$ reduces to the EJSR in the $\Delta s=0$ limit.

To compare the $\chi \mathrm{CQM}$ results with the available experimental data and other model calculations, we can take the case of the quark spin polarizations and the axial coupling constants for the octet baryons at zero momentum transfer. The numerical calculation of the axial-vector coupling constants of the octet baryons at $Q^{2}=0$ involves two set of parameters, the $\mathrm{SU}(3)$ symmetry breaking parameters of $\chi \mathrm{CQM}$ and the mixing angle $\theta$. The 


\begin{tabular}{|c|c|c|c|c|c|}
\hline Parameter $\rightarrow$ & $\phi$ & $a$ & $\alpha$ & $\beta$ & $\zeta$ \\
\hline Value & $20^{\circ}$ & 0.114 & 0.45 & 0.45 & -0.75 \\
\hline
\end{tabular}

TABLE II. Input parameters of the $\chi \mathrm{CQM}$ used in the analysis.

mixing angle $\theta$ is fixed from the consideration of neutron charge radius [41]. The $\chi \mathrm{CQM}$ parameters, $a, a \alpha^{2}, a \beta^{2}$, and $a \zeta^{2}$ represent respectively, the probabilities of fluctuations to pions, $K, \eta$, and $\eta^{\prime}$. A best fit of $\chi \mathrm{CQM}$ parameters can be obtained by carrying out a fine grained analysis of the spin and flavor distribution functions [25, 29] wherein as a first step, a gross analysis was carried out to find the limits of the parameters from the well known experimentally measurable quantities while taking into account strong physical considerations. After obtaining the limits, as a second step, a detailed and fine grained analysis was carried out to obtain the best fit. In Table II, we summarize the input parameters and their values. We would like to mention here that the positive values of $\zeta$ have also been widely used in similar calculations [19]. The sign may not be important for the case of quark spin polarizations in the present context where only $\zeta^{2}$ is involved but since this set of parameters has already been tested for a wide variety of low-energy matrix elements and is able to give a simultaneous fit to the quantities describing proton spin and flavour structure including quark flavor distribution functions (anti quark contents, anti up and anti down quark asymmetry, fraction of quark flavors) as well as the magnetic moments of octet and decuplet baryons etc., we use the same set here. A relative negative sign of $\zeta=c_{1} / c_{8}$ is required primarily to yield the antiquark $\bar{u}-\bar{d}$ asymmetry or the $\bar{u} / \bar{d}$ ratio [47-49] because they involve $\zeta$. The results of the quark spin polarizations and the axial coupling constants for the octet baryons at zero momentum transfer using the parameters listed above have been presented in Table III.

The present experimental situation [11], in terms of the quark spin polarizations, $\Delta u, \Delta d$ and $\Delta s$ for the case of $N$, is summarized as follows:

$$
\begin{aligned}
\Delta u_{N}^{\text {expt }}=0.85 \pm 0.05, \quad \Delta d_{N}^{\text {expt }}=-0.41 \pm 0.05, \quad \Delta s_{N}^{\text {expt }}=-0.07 \pm 0.05 \\
g_{A, N}^{0 \text { expt }}=0.30 \pm 0.06, \quad g_{A, N}^{3 \text { expt }}=1.267 \pm 0.0025, \quad g_{A, N}^{8 \text { expt }}=0.588 \pm 0.033
\end{aligned}
$$

The NQM, which is quite successful in explaining a good deal of low energy data [40,42], 


\begin{tabular}{|c|c|c|c|c|}
\hline Quantity & $N$ & $\Sigma$ & $\Xi$ & $\Lambda$ \\
\hline$\Delta u_{B}$ & 0.904 & 0.881 & -0.329 & 0.002 \\
$\Delta d_{B}$ & -0.362 & -0.137 & 0.00 & 0.002 \\
$\Delta s_{B}$ & -0.023 & -0.252 & 1.109 & 0.805 \\
$g_{A, B}^{0}$ & 0.519 & 0.492 & 0.780 & 0.809 \\
$g_{A, B}^{3}$ & 1.266 & 1.018 & -0.329 & 0.00 \\
$g_{A, B}^{8}$ & 0.588 & 1.248 & -2.547 & -1.606 \\
\hline
\end{tabular}

TABLE III. The $\chi \mathrm{CQM}$ results for the quark spin polarizations and the axial coupling constants for the $N, \Sigma, \Xi$ and $\Lambda$ octet baryons.

has the following predictions for the above mentioned quantities

$$
\begin{aligned}
& \Delta u_{N}=1.33, \quad \Delta d_{N}=-0.33, \quad \Delta s_{N}=0, \\
& g_{A, N}^{0}=1, \quad g_{A, N}^{3}=1.66, \quad g_{A, N}^{8}=1 .
\end{aligned}
$$

The disagreement between the NQM predictions and the DIS measurements was broadly characterized as "proton spin crisis". The results of $\chi \mathrm{CQM}$ for the case of $\Delta u_{N}, \Delta d_{N}, \Delta s_{N}$, $g_{A, N}^{3}$ and $g_{A, N}^{8}$ are more or less in agreement with data. This not only justifies the success of $\chi \mathrm{CQM}$ but also strengthens our conclusion regarding the qualitative and quantitative role of the "quark sea" in right direction. For the case of $g_{A, N}^{0}$, the NQM results show that the valence quarks of the nucleon carry only about $1 / 3$ of the nucleon spin as obtained in the experiment. The $\chi \mathrm{CQM}$ result comes out to be 0.519 which is better than the results of NQM but still shows a large deviation from data. A detailed understanding of the deep inelastic results as well as the dynamics of the constituents of the nucleon constitute a major challenge for any model trying to explain the nonperturbative regime of QCD. In this context, it has been shown recently in a chiral constituent quark potential model that it is possible to describe the singlet axial nucleon coupling if consistent axial exchange currents are taken into account [19, 50, 51]. Because of angular momentum conservation, this reduction of the quark spin is compensated by orbital angular momentum carried by the same nonvalence quark degrees of freedom.

The $Q^{2}$ dependence of the axial-vector form factors have been experimentally investigated from the quasi elastic neutrino scattering [12, 13] and from the pion electroproduction [14]. 
The dipole form of parametrization has been conventionally used to analyse the axial-vector form factors

$$
G_{A, B}^{i}\left(Q^{2}\right)=\frac{g_{A, B}^{i}(0)}{\left(1+\frac{Q^{2}}{M_{A}^{2}}\right)^{2}},
$$

where $g_{A}^{0}(0), g_{A}^{3}(0)$ and $g_{A}^{8}(0)$ are the isovector axial-vector coupling constants at zero momentum transfer. For the axial mass $M_{A}$, a global average as extracted from neutrino scattering experiments is $M_{A}=(1.026 \pm 0.021) \mathrm{GeV}$ [52]. Another recent analysis finds a slightly smaller value $M_{A}=(1.001 \pm 0.020) \mathrm{GeV}[53$. However, in the present work we have used the most recent value obtained by the MiniBooNE Collaboration $M_{A}=1.10_{-0.15}^{+0.13} \mathrm{GeV}$ [54. The axial mass can be taken as free parameter and adjusted to experiment [20. Since experimental data is available only for the nucleon axial coupling constants, we have used the same value of the axial mass for all the octet baryons. The axial masses corresponding to $\Sigma, \Xi$ and $\Lambda$ are expected to be larger than that of the nucleon which will in turn lead to slightly larger values of the axial-vector form factors in magnitude. The overall behavior of the form factors however will not be affected by this change.

After having incorporated $Q^{2}$ dependence in the axial-vector form factors, we now discuss the variation of all the $Q^{2}$ dependent quantities in the range $0 \leq Q^{2} \leq 1$. In Fig. 1, we have presented the singlet and non-singlet axial-vector form factors of the octet baryons $N$, $\Sigma, \Xi$ and $\Lambda$. From a cursory look at the plots, one can easily describe some general aspects of the sensitivity to $Q^{2}$ for the form factors. The sensitivity of the singlet and non-singlet form factors for different baryons varies as

$$
\begin{aligned}
& G_{A, \Xi}^{0}>G_{A, \Lambda}^{0}>G_{A, N}^{0}>G_{A, \Sigma}^{0}, \\
& G_{A, N}^{3}>G_{A, \Sigma}^{3}>G_{A, \Xi}^{3}>G_{A, \Lambda}^{3}, \\
& G_{A, \Xi}^{8}>G_{A, \Lambda}^{8}>G_{A, N}^{8}>G_{A, \Sigma}^{8} .
\end{aligned}
$$

The behaviour of the form factors for $\Xi$ and $\Lambda$ is similar to each other. This may possibly due to the presence of more strange quarks in the valence structure. On the other hand, the form factors for $N$ and $\Sigma$, which have the dominance of $u$ quarks in the valence structure, show similar variation with $Q^{2}$. This can be easily seen from Fig. 1 and this is true for $G_{A, B}^{0}$, $G_{A, B}^{3}$ as well as $G_{A, B}^{8}$. Another important observation for the case of $G_{A, B}^{0}$ form factors is that it falls off rapidly with the increase of $Q^{2}$ for all the octet baryons $N, \Sigma, \Xi$ and $\Lambda$. However, for the case of $G_{A, B}^{3}$ and $G_{A, B}^{8}$, the $N$ and $\Sigma$ form factors fall off with increasing 

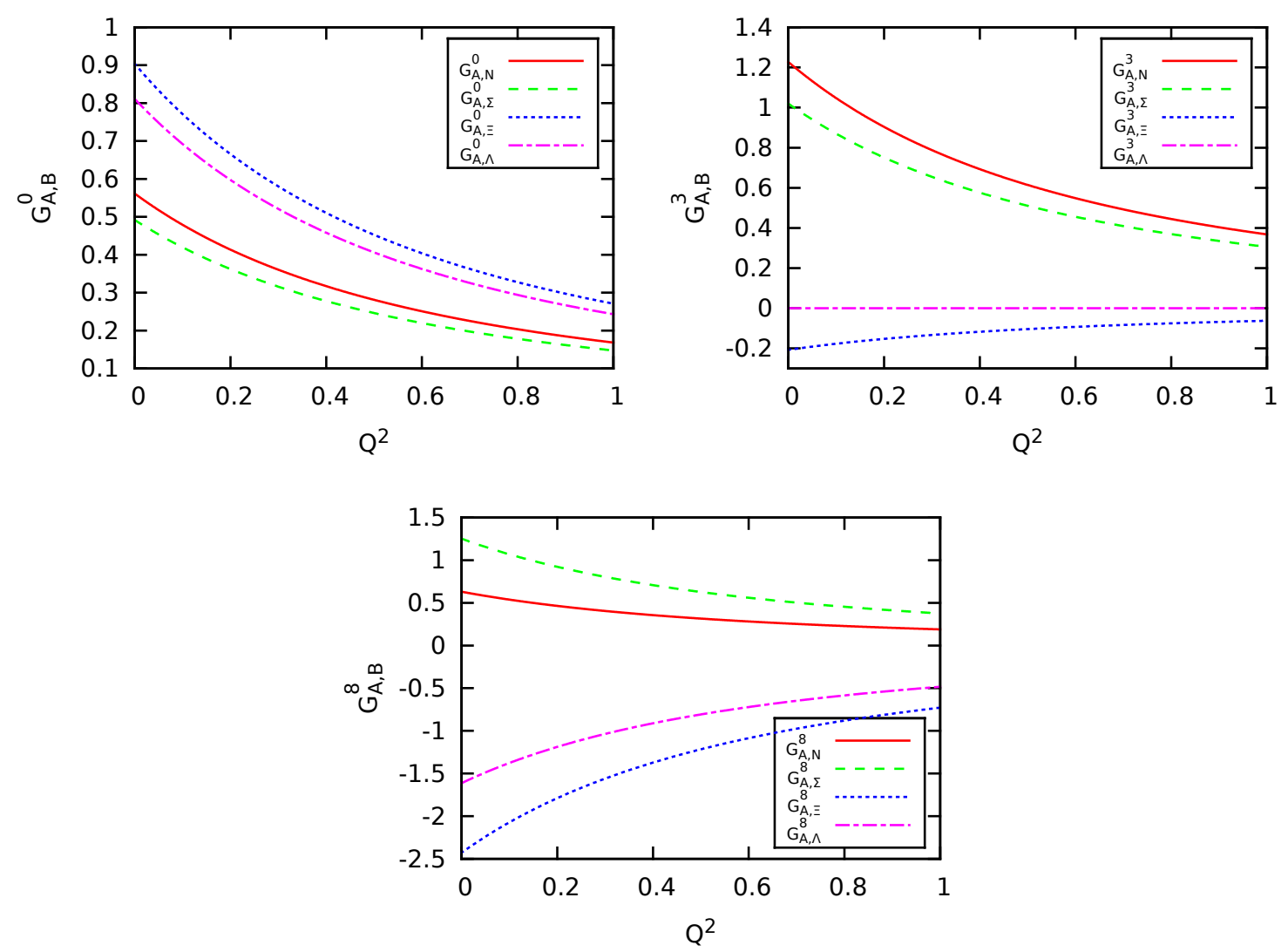

FIG. 1. (color online). Form factors for the baryons $N, \Sigma, \Xi, \Lambda$ plotted as function of $Q^{2}$.

$Q^{2}$ whereas the $\Xi$ and $\Lambda$ form factors increase with increasing $Q^{2}$. The case of $G_{A, \Lambda}^{3}$ is particularly interesting because of its flavor structure which has equal numbers of $u, d$, and $s$ quarks in its valence structure. Unlike the other octet baryons, where the form factors decrease or increase continuously with the $Q^{2}$ values, the form factor in this case has no $Q^{2}$ dependence.

Since the constituent quarks are spatially extended particles [20, 55], they themselves have axial form factors. The role of non-valence quarks in the spin structure can be studied in detail by calculating the flavor axial-vector form factors using the dipole form of parametrization (Eq. (29). These can be expressed in terms of the singlet and non-singlet combinations of the spin structure as follows

$$
\begin{aligned}
G_{A, B}^{u} & =\frac{1}{3} G_{A, B}^{0}+\frac{1}{2} G_{A, B}^{3}+\frac{1}{2 \sqrt{3}} G_{A, B}^{8}, \\
G_{A, B}^{d} & =\frac{1}{3} G_{A, B}^{0}-\frac{1}{2} G_{A, B}^{3}+\frac{1}{2 \sqrt{3}} G_{A, B}^{8},
\end{aligned}
$$



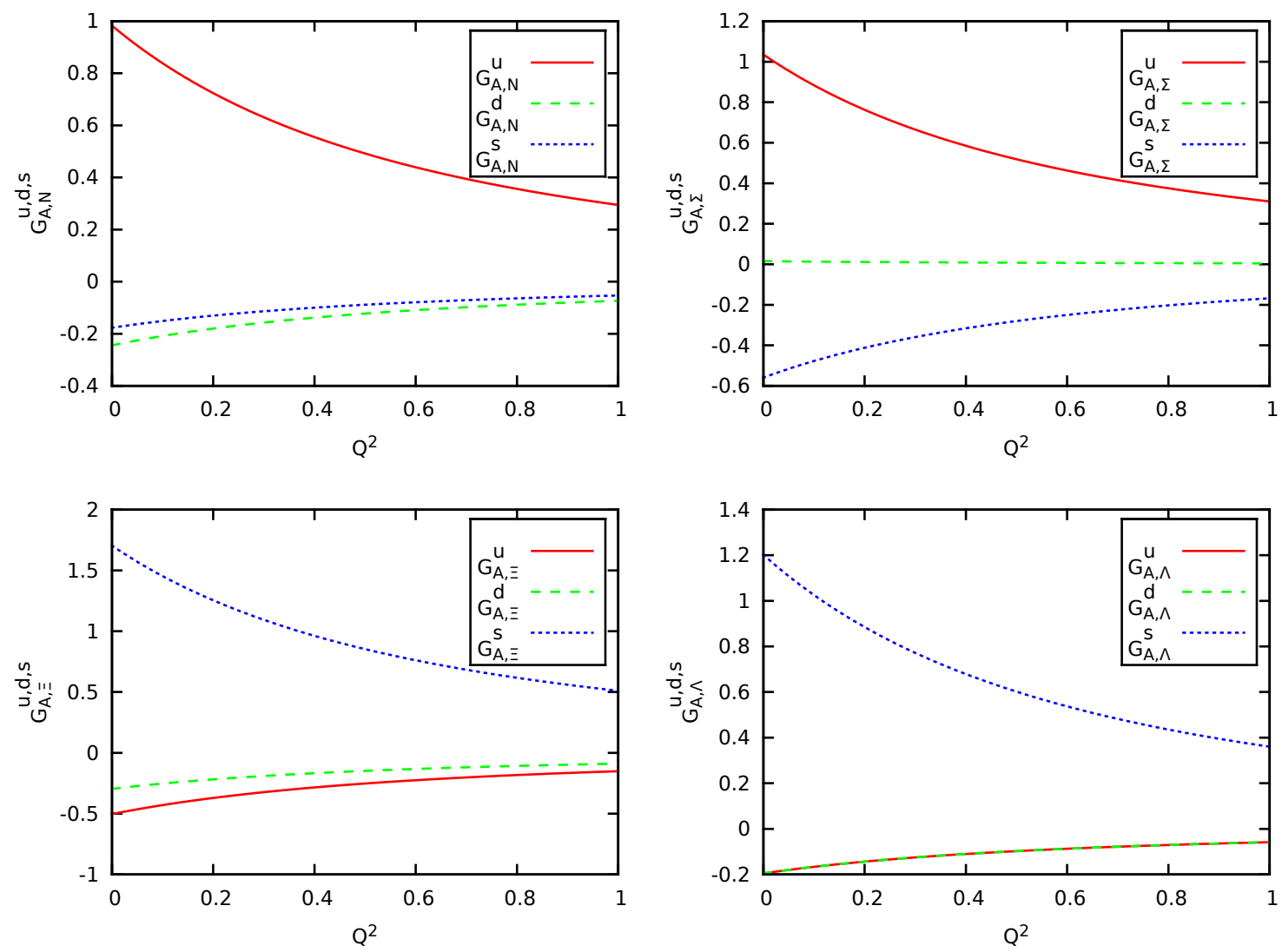

FIG. 2. (color online). The explicit flavor form factors for the baryons $N, \Sigma, \Xi, \Lambda$ plotted as function of $Q^{2}$.

$$
G_{A, B}^{s}=\frac{1}{3} G_{A, B}^{0}-\frac{1}{\sqrt{3}} G_{A, B}^{8}
$$

In Fig. 2, we have plotted the explicit $u, d$, and $s$ quark flavor contributions for each of the octet baryon axial-vector form factors. The plots clearly project out the valence quark structure of the baryon. For example, since $N$ is dominated by $u$ quark it is clear from the plot of $G_{A, N}^{u, d, s}$ that the $G_{A, N}^{u}$ dominates and $G_{A, N}^{d}, G_{A, N}^{s}$ has a comparatively smaller contribution. The important observation in this case is the non-zero contribution of the $s$ quarks. Even though there are no $s$ quarks in the valence structure the contribution of $G_{A, N}^{s}$ implies a presence of "quark sea" which is even more at zero momentum transfer. It is also evident from the figure that the valence quark distribution is spread over the entire $Q^{2}$ region and as the value of $Q^{2}$ increases, the sea contributions decrease and at even higher values of $Q^{2}$ (not presented here), the contributions should be completely dominated by the valence quarks. Further, for the case of $G_{A, \Sigma}^{u, d, s}$ and $G_{A, \Xi}^{u, d, s}$, where the valence structure is 


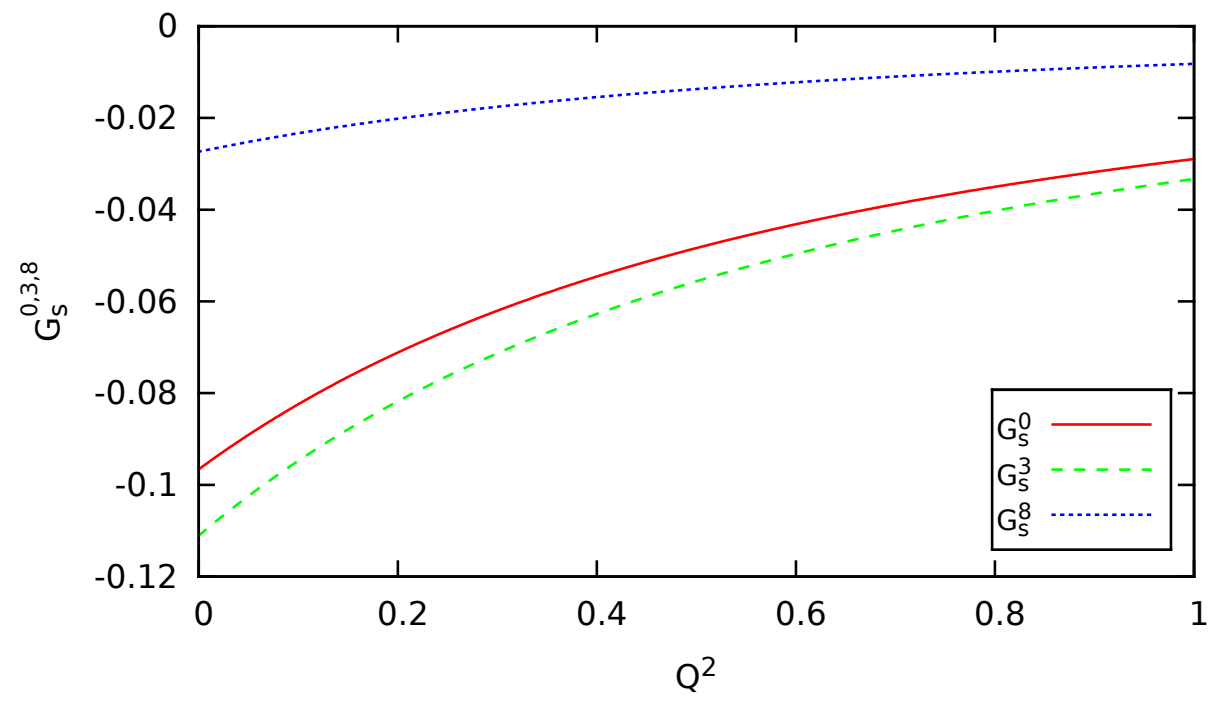

FIG. 3. (color online). The strange form factors for the nucleon plotted as function of $Q^{2}$.

dominated by the $u$ and $s$ quarks, we find a significant contribution from them. In these form factors, the small but significant $G_{A}^{d}$ can have important implications for the role of sea quarks at low $Q^{2}$. Finally, the $G_{A, \Lambda}^{u, d, s}$, even after having equal contributions from the $u$, $d$, and $s$ quarks, does not show a symmetric behaviour. The $G_{A, \Lambda}^{s}$ clearly dominates over $G_{A, \Lambda}^{u}$ and $G_{A, \Lambda}^{d}$ which is expected because of the $u$ and $d$ quarks also contribute towards $G_{A, \Lambda}^{u, d, s}$ through quark fluctuations. It is interesting to note that the valence and sea quark distributions contribute in the right direction to give an excellent overall fit to the axialvector form factors where experimental data is available. This can perhaps be substantiated further by a measurements for the other octet baryons.

It is well known that, for the case of nucleon, the strange quarks contribute to the spin polarizations of $u$ and $d$ quarks apart from contributing to the strange spin polarization. This is because of the presence of the non-valence "quark sea" (Eq. (2)). In this context, the axial-vector matrix elements will have implications for the strangeness contribution to the nucleon as well as for the effects of chiral symmetry breaking. We can calculate $G_{s}^{0}\left(Q^{2}\right)$, $G_{s}^{3}\left(Q^{2}\right)$ and $G_{s}^{8}\left(Q^{2}\right)$ for the case of $N$ from Eq. (26) and Table I by dropping the constant factors. The factors with $a \alpha^{2}, a \beta^{2}$ and $a \zeta^{2}$ include the effects of chiral symmetry breaking as well as $\mathrm{SU}(3)$ symmetry breaking and give the contribution coming from the "quark sea". In particular, they give the contribution of strange quarks to the nucleon spin. The explicit strangeness contribution for the other octet baryons is not so significant because of 


\begin{tabular}{|c|c|c|c|c|c|}
\hline Quantity $\rightarrow$ & $\Delta u_{s}$ & $\Delta d_{s}$ & $g_{s}^{0}$ & $g_{s}^{3}$ & $g_{s}^{8}$ \\
\hline $\mathrm{NQM}$ & 0 & 0 & 0 & 0 & 0 \\
$\chi \mathrm{CQM}$ & -0.092 & 0.013 & -0.102 & -0.105 & -0.033 \\
\hline
\end{tabular}

TABLE IV. The NQM and $\chi \mathrm{CQM}$ results for the explicit strangeness contribution to spin polarizations and the axial coupling constants at $Q^{2}=0$ for the case of $N$.

the presence of strange quarks in their valence structure. In Fig. 3, we have presented the results for $G_{s}^{0}\left(Q^{2}\right), G_{s}^{3}\left(Q^{2}\right)$ and $G_{s}^{8}\left(Q^{2}\right)$ for the case of $N$. We find that the magnitude of $G_{s}^{0}\left(Q^{2}\right)$ and $G_{s}^{8}\left(Q^{2}\right)$ fall off with the increasing value of $Q^{2}$ whereas $G_{s}^{3}\left(Q^{2}\right)$ has a weak $Q^{2}$ dependence. For the sake of completeness we have also presented the numerical values of the explicit strangeness contribution to $\Delta u, \Delta d$ and the axial coupling constants at $Q^{2}=0$ for the case of $N$ in Table IV. The contribution of $\Delta s$ is coming purely from the quark sea and has already been presented in Table III. It is clear from the table that there is a significant contribution of non-valence quarks in $\Delta u_{s}, g_{s}^{0}$ and $g_{s}^{3}$. These quantities not only provide a direct method to determine the presence of a significant amount of quark sea but also impose important constraint on a model that attempts to describe the origin of the quark sea. A small but significant contribution of strangeness in the nucleon has already been indicated by SAMPLE at MIT-Bates [7], G0 at JLab 8], PVA4 at MAMI [9] and HAPPEX at JLab [10]. A determination of $G_{A}^{s}$ at low values of $Q^{2}$ [54] would permit a determination of strange spin polarization $\Delta s$ which is otherwise zero in the case of nucleon. The strange quarks contribute through the quark sea generated by the chiral fluctuations and any refinement in the case of the strangeness dependent quantities would have important implications for the basic tenets of $\chi \mathrm{CQM}$.

To summarize, the chiral constituent quark model $(\chi \mathrm{CQM})$ is able to phenomenologically estimate the quantities having implications for chiral symmetry breaking and SU(3) symmetry breaking. In particular, it provides a fairly good description of the axial-vector form factors of the low lying octet baryons $(N, \Sigma, \Xi$ and $\Lambda)$, for example, the singlet $\left(g_{A}^{0}\right)$ and non-singlet $\left(g_{A}^{3}\right.$ and $\left.g_{A}^{8}\right)$ axial-vector coupling constants expressed as combinations of the spin polarizations at zero momentum transfer. In order to enlarge the scope of $\chi \mathrm{CQM}$, we have used the conventional dipole form of parametrization to analyse the $Q^{2}$ dependence of the axial-vector form factors $\left(G_{A}^{0}\left(Q^{2}\right), G_{A}^{3}\left(Q^{2}\right)\right.$ and $\left.G_{A}^{8}\left(Q^{2}\right)\right)$. To understand the role of 
chiral symmetry breaking and the significance of non-valence quarks in the nucleon structure, the implications of hidden strangeness component have been studied for the strange singlet and non-singlet contents $\left(G_{s}^{0}\left(Q^{2}\right), G_{s}^{3}\left(Q^{2}\right)\right.$ and $\left.G_{s}^{8}\left(Q^{2}\right)\right)$ of the nucleon. The $\chi \mathrm{CQM}$ is able to give a qualitative and quantitative description of the axial-vector form factors. The significant contribution of the strangeness is also consistent with the recent available experimental results.

In conclusion, we would like to state that chiral symmetry breaking and $\mathrm{SU}(3)$ symmetry breaking play an important role in understanding the spin structure of the baryon and is the key to describe the hidden strangeness content of the nucleon in the nonperturbative regime of QCD where the constituent quarks and the weakly interacting Goldstone bosons constitute the appropriate degrees of freedom at the leading order. The future experiments to measure the axial-vector form factors will not only provide a direct method to determine

the presence of appropriate amount of quark sea but also impose important constraint on the parity-violating asymmetries in different kinematical regions. Several groups, for example, Miner $\nu$ a are contemplating the possibility of performing the high precision measurements over a wide $Q^{2}$ region in the near future.

\section{ACKNOWLEDGMENTS}

HD would like to thank Department of Science and Technology, Government of India for financial support.

[1] J. Ashman et al. (EMC Collaboration), Phys. Lett. B 206, 364 (1988); Nucl. Phys. B 328, 1 (1989).

[2] B. Adeva et al. (SMC Collaboration), Phys. Rev. D 58, 112001 (1998).

[3] P. Adams et al., Phys. Rev. D 56, 5330 (1997); P.L. Anthony et al. (E142 Collaboration), Phys. Rev. Lett. 71, 959 (1993); K. Abe et al. (E143 Collaboration), Phys. Rev. Lett. 76, 587 (1996); K. Abe et al. (E154 Collaboration), Phys. Rev. Lett. 79, 26 (1997).

[4] A. Airapetian et al. (HERMES Collaboration), Phys. Rev. D 71, 012003 (2005).

[5] J.D. Bjorken, Phys. Rev. 148, 1467 (1966); Phys. Rev. D 1, 1376 (1970).

[6] J. Ellis and R.L. Jaffe, Phys. Rev. D 9, 1444 (1974); ibid. 10, 1669 (1974). 
[7] D.T. Spayde et al. (SAMPLE Collaboration), Phys. Lett. B 583, 79 (2004).

[8] D. Armstrong et al. (G0 Collaboration), Phys. Rev. Lett. 95, 092001 (2005). D. Androić et al. (G0 Collaboration), Phys. Rev. Lett. 104, 012001 (2010).

[9] F.E. Maas et al. (PVA4 Collaboration), Phys. Rev. Lett. 93, 022002 (2004); F.E. Maas et al. (PVA4 Collaboration), Phys. Rev. Lett. 94, 152001 (2005).

[10] K.A. Aniol et al. (HAPPEX Collaboration), Phys. Rev. C 69, 065501 (2004); K.A. Aniol et al. (HAPPEX Collaboration), Phys. Rev. Lett. 98, 032301 (2007); K.A. Aniol et al. (HAPPEX Collaboration), Eur. Phys. J. A 31, 597 (2007); Z. Ahmed et al. (HAPPEX Collaboration), arXiv:1107.0913v1 [nucl-ex].

[11] J. Beringer et al. (Particle Data Group), Phys. Rev. D 86, 010001 (2012).

[12] T. Kitagaki et al., Phys. Rev. D bf 28, 436 (1983).

[13] L.A. Ahrens et al., Phys. Rev. D 35, 785 (1987); L. A. Ahrens et al., Phys. Lett. B 202, 284 (1988).

[14] S. Choi et al., Phys. Rev. Lett. 71, 3927 (1993); A. Liesenfeld et al. (A1 Collaboration), Phys. Lett. B bf 468, 20 (1999).

[15] Howard Scott Budd, A. Bodek, J. Arrington, Nucl. Phys. Proc. Suppl. 139, 90 (2005).

[16] R.G. Edwards et al. (LHPC), Phys. Rev. Lett. 96, 052001 (2006); A.A. Khan et al., Phys. Rev. D 74, 094508 (2006); C. Alexandrou, G. Koutsou, T. Leontiou, J. W. Negele, and A. Tsapalis, Phys. Rev. D 76, 094511 (2007); P. Hagler et al., Phys. Rev. D 77, 094502 (2008); T. Yamazaki et al. (RBC+UKQCD), Phys. Rev. Lett. 100, 171602 (2008).

[17] M. Casu and L.M. Sehgal, Phys. Rev. D 58, 033002 (1998); Antonio Silva, Hyun-Chul Kim, Diana Urbano and Klaus Goeke, Phys. Rev. D 72, 094011 (2005); Stephen F. Pate, Glen A. MacLachlan, David W. McKee and Vassili Papavassiliou, AIP Conf. Proc. 842, 309 (2006); M.R. Schindler and S. Scherer, Eur. Phys. J. A 32, 429 (2007); Guray Erkol and Altug Ozpineci, Phys. Rev. D 83, 114022 (2011); Stephen Pate and Dennis Trujillo, EPJ Web Conf. bf 66,06018 (2014).

[18] D. Barquilla-Cano, A.J. Buchmann, E. Hernandez, Nucl. Phys. A 714, 611 (2003).

[19] D. Barquilla-Cano, A.J. Buchmann, E. Hernandez, Eur. Phys. J. A 27, 365 (2006).

[20] D. Barquilla-Cano, A.J. Buchmann, E. Hernandez, Phys. Rev. C 75, 065203 (2007).

[21] S. Weinberg, Physica A 96, 327 (1979); A. Manohar and H. Georgi, Nucl. Phys. B 234, 189 (1984); E.J. Eichten, I. Hinchliffe, and C. Quigg, Phys. Rev. D 45, 2269 (1992). 
[22] T.P. Cheng and L.F. Li, Phys. Rev. Lett. 74, 2872 (1995); Phys. Rev. D 57, 344 (1998); Phys. Rev. Lett. 80, 2789 (1998).

[23] J. Linde, T. Ohlsson, and H. Snellman, Phys. Rev. D 57, 452 (1998); 57, 5916 (1998).

[24] X. Song, J.S. McCarthy, and H.J. Weber, Phys. Rev. D 55, 2624 (1997); X. Song, Phys. Rev. D 57, 4114 (1998).

[25] H. Dahiya and M. Gupta, Phys. Rev. D 64, 014013 (2001); H. Dahiya and M. Gupta, Int. Jol. of Mod. Phys. A, Vol. 19, No. 29, 5027 (2004); H. Dahiya, M. Gupta and J.M.S. Rana, Int. Jol. of Mod. Phys. A, Vol. 21, No. 21, 4255 (2006).

[26] H. Dahiya and M. Gupta, Phys. Rev. D 66, 051501(R) (2002); 67, 114015 (2003).

[27] H. Dahiya and M. Gupta, Eur. Phys. J. C 52, 571 (2007); N. Sharma and H. Dahiya, Phys. Rev. D 81, 114003 (2010).

[28] N. Sharma, H. Dahiya, P.K. Chatley, and M. Gupta, Phys. Rev. D 79, 077503 (2009); N. Sharma, H. Dahiya, and P.K. Chatley, Eur. Phys. J. A 44, 125 (2010).

[29] H. Dahiya and M. Gupta, Phys. Rev. D 78, 014001 (2008); H. Dahiya and N. Sharma, Mod. Phys. Lett. A, Vol. 26, No. 4, 279 (2011).

[30] N. Sharma, A.M. Torres, K.P. Khemchandani, and H. Dahiya, Eur. Jol. Phys. A 49, 11 (2013).

[31] A.M. Torres, K.P. Khemchandani, N. Sharma, and H. Dahiya, Eur. Jol. Phys. A 48, 185 (2012).

[32] N. Sharma and H. Dahiya, Pramana, Journal of Physics, Volume 81, Issue 3, 449 (2013).

[33] N. Sharma and H. Dahiya, Pramana, 80, Issue 2, 237-249 (2013).

[34] H. Dahiya and M. Gupta, Phys. Rev. D 67, 074001 (2003).

[35] N. Sharma, H. Dahiya, P.K. Chatley, and M. Gupta Phys. Rev. D 81, 073001 (2010).

[36] N. Sharma and H. Dahiya, Int. Jol. of Mod. Phys. A, Vol. 28, No. 14, 1350052 (2013).

[37] L.Ya. Glozman and D.O. Riska, Phys. Rep. 268, 263 (1996), L.Ya. Glozman, Z. Papp and W. Plessas, Phys. Lett. 381B, 311 (1996).

[38] T.P. Cheng and Ling Fong Li, hep-ph/9811279.

[39] Adam P. Szczepaniak and Erie S. Swanson Phys. Rev. Lett. 87, 072001 (2001).

[40] N. Isgur, G. Karl and R. Koniuk, Phys. Rev. Lett. 41, 1269 (1978); N. Isgur and G. Karl, ibid. , 3175 (1980); N. Isgur et al., ibid. 35, 1665 (1987); P. Geiger and N. Isgur, ibid. 55, 299 (1997).

[41] A. De Rujula, H. Georgi and S.L. Glashow, Phys. Rev. D 12, 147 (1975). 
[42] A. Le Yaouanc, L. Oliver, O. Pene and J.C. Raynal, Phys. Rev. D 15, 844 (1977).

[43] P.N. Pandit, M.P. Khanna and M. Gupta, J. Phys. G 11, 683 (1985).

[44] M. Gupta and N. Kaur, Phys. Rev. D 28, 534 (1983).

[45] M. Gupta, J. Phys. G 16, L213 (1990).

[46] A. Le Yaouanc et al., Hadron Transitions in the Quark Model, Gordon and Breach, New York, (1988).

[47] New Muon Collaboration, P. Amaudruz et al., Phys. Rev. Lett. 66, 2712 (1991); M. Arneodo et al., Phys. Rev. D 50, R1 (1994).

[48] NA51 Collaboration, A. Baldit et al., Phys. Lett. 253B, 252 (1994).

[49] E866/NuSea Collaboration, E.A. Hawker et al., Phys. Rev. Lett. 80, 3715 (1998); J.C. Peng et al., Phys. Rev. D 58, 092004 (1998); R. S. Towell et al., ibid. 64, 052002 (2001).

[50] D. Barquilla-Cano, E. Hernandez and A.J. Buchmann Nucl. Phys. A 721, 429 (2003); A.J. Buchmann and E.M. Henley, Phys. Rev. D 83, 096011 (2011).

[51] P.E. Shanahan, A.W. Thomas, K. Tsushima, R.D. Young and F. Myhrer Phys. Rev. Lett. 110, 202001 (2013).

[52] V. Bernard, L. Elouadrhiri and U.-G. Meiner, J. Phys. G 28, R1 (2002).

[53] H. Budd, A. Bodek and J. Arrington, Nucl. Phys. Proc. Suppl. 139, 90-95 (2005).

[54] A.A. Aguilar-Arevalo et al. (MiniBooNE Collaboration), Phys. Rev. D 82, 092005 (2010); Tomasz Golan, Krzysztof M. Graczyk, Cezary Juszczak, Jan T. Sobczyk, Phys. Rev. C 88, $024612(2013)$.

[55] A.J. Buchmann, E. Hernandez, and Amand Faessler, Phys. Rev. C 55, 448 (1997); A.J. Buchmann, E. Hernandez, U. Meyer and A. Faessler, Phys. Rev. C 58, 2478 (1998); U. Meyer, E. Hernandez and A.J. Buchmann, Phys. Rev. C 64, 035203 (2001). 